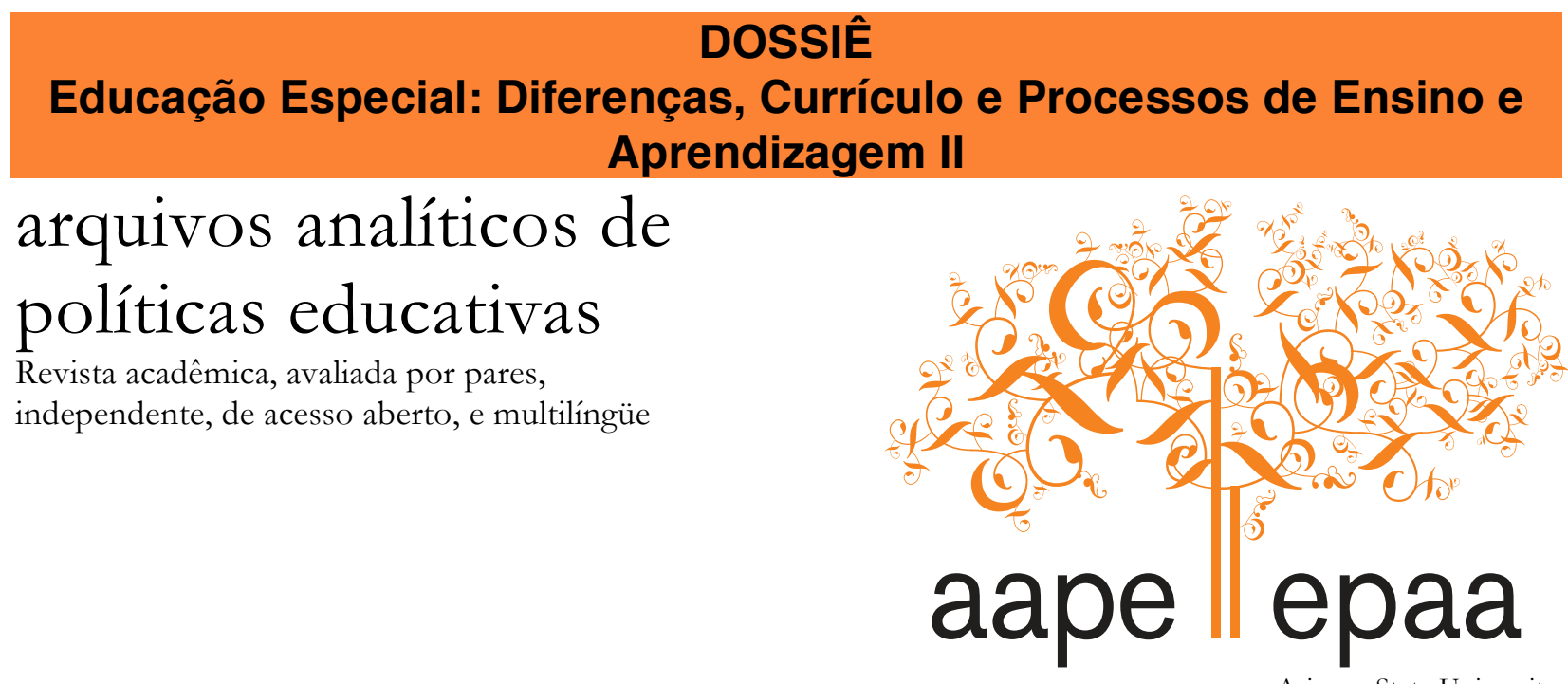

Arizona State University

\title{
Avaliação da Condição de Alfabetização de Estudantes com Deficiência Intelectual no Contexto Inclusivo
}

\author{
Anna Augusta Sampaio de Oliveira \\ Programa de Pós-graduação em Educação \\ UNESP, Marília/SP \\ Brasil
}

Citação: Oliveira, A. A. S. (2015). Avaliação da condição de alfabetização de estudantes com Deficiência Intelectual no contexto inclusivo. Arquivos Analíticos de Políticas Educativas, 23(31). Dossiê Educação Especial: Diferenças, Currículo e Processos de Ensino e Aprendiragem II. Editoras convidadas: Márcia Denise Pletsch \& Geovana Mendonça Lunardi Mendes. http://dx.doi.org/10.14507/epaa.v23.1627

Resumo: A questão da avaliação na área da deficiência intelectual, numa perspectiva inclusiva, ainda é tema pouco explorado na pesquisa e na prática educacional. Neste estudo apresentamos resultado de investigação sobre o desempenho na fase inicial de alfabetização. Foram avaliados 32 estudantes com deficiência intelectual, do $2^{\circ}$ ano do ensino fundamental, de uma das regiões centrais do município de São Paulo. Os resultados apontam possibilidades de aprendizagem como também as dificuldades na área da leitura e escrita destes estudantes.

Palavras-chave: alfabetização; deficiência intelectual; avaliação.

\section{Assessment of Students with Intellectual Disabilities Literacy Condition in Inclusive Context}

Abstract: The issue of evaluation in the area of intellectual disabilities, an inclusive perspective, is still relatively unexplored in research and educational practice. This study presents results of research on the performance in early literacy. We evaluated 32 students with intellectual disabilities, the 2nd 
year of elementary school, one of the central regions of São Paulo. The results show learning opportunities as well as difficulties in the area of reading and writing of these students.

Keywords: alphabetization; intellectual disabilities; assessment.

\section{Evaluación de las Condiciones la Alfabetización los Estudiantes con Discapacidad Intelectual en Contexto Inclusivo}

Resumen: El tema de la evaluación en el ámbito de la discapacidad intelectual en una perspectiva inclusiva, es aún tema poco explorado en la investigación y la práctica educativa.

Este estudio presenta los resultados de la investigación sobre el desempeño en la alfabetización temprana. 32 estudiantes con discapacidad intelectual, el segundo año de la escuela primaria, una de las regiones centrales de São Paulo, fueron evaluados. Los resultados indican oportunidades de aprendizaje, así como dificultades en el área de la lectura y la escritura de estos estudiantes. Palabras-clave: alfabetización; discapacidad intelectual; evaluación.

\section{Introdução}

Não são poucos e nem pequenos os entrelaçamentos quando nos propomos a discutir alfabetização. Por si só este tema bastaria, visto sua complexidade e os vários fatores intervenientes no processo de escrita e de leitura. Indiscutivelmente é um tema difícil, complexo, de múltipla análise que nos desafia o tempo todo: aos professores e professoras, aos universitários e universitárias, aos pesquisadores e pesquisadoras, aos pais (pais e mães), a escola e até mesmo a política nacional. Alfabetizar. Meta maior, desejo imperioso de todos que estão envolvidos nesta problemática.

A alfabetização se configura como um importante problema de política pública. Não à toa, frente aos dados nacionais de desempenho em leitura e escrita das crianças brasileiras, é a alfabetização, realmente, um problema real. Mas, não é só isto que queremos abordar. Há, também, a questão da inclusão escolar como política pública anunciada no desejo de constituição de uma escola para todos - plural, democrática, inclusiva! Redundância, certamente, pois ao falar em escola já deveríamos compreendê-la como plural, democrática, inclusiva e, principalmente, para todos. E todos seriam mesmo todos! Então, introduzimos outro ponto de nosso debate: a deficiência intelectual, como parte deste todo anunciado pela escola.

É o assunto é multiplamente complexo: inclusão escolar, alfabetização, deficiência intelectual. No entanto, é necessário que a pesquisa pedagógica também se ocupe deste tema e é este o objetivo do presente artigo.

\section{Notas Teóricas}

O ideário de inclusão escolar presente na legislação nacional (Brasil, 1996, 2001, 2003, 2010a, 2010b, 2010c, 2011a, 2011b), e nos debates de vários autores contemporâneos (Glat, 2007; Jesus et al., 2007; Oliveira et al., 2008; Pacheco et al., 2007; Pletsch, 2010) nos coloca frente a implicações profundas no contexto escolar. Ao falar de uma proposta inclusivista, indubitavelmente, falamos de uma nova escola ou de uma escola que se renove e que busque superar a exclusão cotidiana presente em sua organização e em suas práticas. Este é um ponto importante: falar de inclusão escolar traz implicações para escola brasileira; indiscutivelmente! Não é possível desconsiderar as diferenças impostas pela diversidade e nem tão pouco considerar a deficiência como mera diferença (Omote, 1996, 2008), uma vez que ela impõe a necessidade de se buscar as "vias colaterais" do desenvolvimento, no sentido descrito por Vygotski:

As vias colaterais veem a ser como um experimento espontâneo da natureza e demonstram que o desenvolvimento cultural da conduta não está obrigatoriamente 
relacionado com uma ou outra função orgânica. Não é obrigatório que a linguagem dependa do aparato fônico, pode encarnar-se em outro sistema de signos, assim como a escrita de sistema visual pode transformar-se em um sistema tátil.[...] Dessa maneira se criam vias colaterais de desenvolvimento onde resulta a impossibilidade da via direta. (2000, p. 311) (tradução livre)

Isto nos anuncia o papel preponderante que a escola deve exercer para impulsionar o desenvolvimento daqueles com deficiência, para que possa compensar os "defeitos" primários - de origem biológica (Vygotki, 1997), com vias colaterais a serem utilizadas no processo de ensino. Grande desafio, uma vez que se coloca uma função indispensável para a escola: intervir no desenvolvimento humano e, na perspectiva inclusiva, no contexto da diversidade.

Embora estejamos avançando na constituição de uma escola que acolhe e procura manter aqueles com deficiência em seu contexto, infelizmente, ainda temos cometido muitas impropriedades na condução deste processo no cotidiano pedagógico e, muitas vezes, as pessoas com deficiência intelectual apenas ocupam um lugar, sem a garantia da aprendizagem. Para exemplificar, podemos utilizar a questão da alfabetização (ou a ausência dela). Quantos estão na escola e se alfabetizam? Como a escola tem feito para garantir essa aprendizagem?

A maioria desses sujeitos, vítima de grande preconceito, pouco tem conseguido avançar em seu processo de escolarização [...] e, quando o consegue, na maioria das vezes, os conhecimentos adquiridos não correspondem ao trabalho naquela série/nível de ensino. É o caso de inúmeros estudantes que têm chegado ao segundo segmento do Ensino Fundamental [...] analfabetos ou semialfabetizados, com dificuldades acentuadas na escrita, depois de uma trajetória difícil nos anos iniciais de escolarização. (Iacono, 2012, p.94)

As justificativas frente à problemática apresentada por Iacono são muitas e, quase sempre, localizadas na deficiência e na individualidade como fontes de impedimento para aprendizagem. Não há como não se indignar frente a essa posição passiva e confortável de colocar no sujeito e em sua deficiência as causas da não aprendizagem.

Oliveira (2010) ao estudar a questão da apropriação da escrita por estudantes com Síndrome de Down, aponta suas possibilidades de aprendizagem e a importância fundamental da mediação pedagógica como forma de impulsionar o desenvolvimento destes estudantes em direção à escrita. Diz a autora:

A análise dos dados apontou que o envolvimento das crianças com o processo de escrita se ampliou significativamente, de modo que pudemos observar, cada vez mais, uma aproximação com o código linguístico, principalmente de duas delas, as quais apresentavam um maior distanciamento e desinteresse na produção escrita. Foram verificados avanços nas representações gráficas e nas tentativas individuais de escrita. (p. 353)

Ferreira (2007) apresenta uma argumentação em favor do letramento dos estudantes com deficiência intelectual como base de adequação curricular para o trabalho em classes comuns do ensino regular. Monteiro (2004) também argumenta a favor da possibilidade de escrita daqueles com deficiência intelectual e, inclusive, questiona se a não aprendizagem de conteúdos de escrita não estaria ligada as oportunidades (ou a falta delas) na vida escolar destes estudantes. Saad (2003) apresenta um trabalho, com base na Teoria Histórico-cultural, no qual apresenta as possibilidades de escrita daqueles com deficiência intelectual. Machado e Marquezan (2003) discutem as possibilidades da escrita na área da deficiência intelectual, através do texto jornalístico e Bonetti (1999), Ide (1992, 1993), Mantoan (1989) possuem trabalhos que apontam a constituição da base alfabética de escrita de estudantes com deficiência intelectual, principalmente aqueles com Síndrome de Down. 
No entanto, apesar de alguns estudos já apontaram as possibilidades de escrita destes estudantes, alguns autores como Braun (2012), Pletsch (2010), Padilha, Oliveira e Silva (2014, no prelo) apontam a questão da prática pedagógica voltada para o estudante com deficiência intelectual, comentando sobre os desencontros, as propostas e sobre, inclusive, a postura do professor e o quanto ele mesmo é ainda pouco respeitado e compreendido em sua inserção pedagógica numa perspectiva inclusiva e, muitas vezes, não possui conhecimento específico para lidar com as necessidades educacionais especiais provenientes da deficiência intelectual.

Por outro lado, temos que questionar como estão sendo avaliados estes estudantes e suas condições de aprendizagem? Quais são os instrumentos de avaliação utilizados para acompanhar este processo naqueles com deficiência intelectual?

Em alguns estudos realizados os resultados sobre a aprendizagem curricular desses estudantes acabam por apontar dificuldades expressivas em suas competências na área da leitura e da escrita (Valentim, 2011; Oliveira, 2012a,2012b, 2011), interpondo à produção científica um grande desafio: o processo de alfabetização daqueles com deficiência intelectual. Esse se configura como um problema de pesquisa que exige maior número de investigações, em busca de caminhos para o ensino da leitura e da escrita por aqueles com deficiência intelectual.

\section{Notas Sobre a Pesquisa}

O estudo apresenta um recorte de pesquisa realizada no município de São Paulo tendo como objeto a avaliação do desempenho escolar de 1.422 estudantes com deficiência intelectual (DI), por meio de instrumento de avaliação utilizado pela rede municipal de educação, elaborado sob nossa supervisão: o Referencial de Avaliação da Aprendizagem na Área da Deficiência Intelectual - Ensino Fundamental I - o RAADI. ${ }^{1}$

Para fins deste trabalho serão apresentados dados de Língua Portuguesa, tendo como referência o RAADI, de 32 estudantes com DI, matriculados no $2^{\circ}$ ano do ensino fundamental de 9 anos, de uma das regiões centrais da capital paulista. Esta região conta com um total de 32 escolas de Ensino Fundamental nas quais foi utilizado o RAADI como instrumento de avaliação e em 20 delas se encontram os 32 estudantes com DI que frequentam o $2^{\circ}$ ano e foram avaliados.

Trata-se de uma pesquisa descritiva, uma vez que, em conformidade com o expresso por Gil (2002), descreve as características de aprendizagem na área da Língua Portuguesa, de um grupo específico - aqueles com DI - com base nos registros de desempenho escolar avaliado pelas professoras, com a utilização de um instrumento previamente construído - o RAADI - que, como mencionado, caracteriza-se como um referencial de avaliação dos conteúdos curriculares na área em estudo.

A coleta de dados baseou-se nos documentos de avaliação semestral de cada um dos 32 estudantes, a partir dos registros das professoras das salas comuns por eles frequentadas. Elas utilizaram o RAADI e os dados referem-se aos indicadores de aprendizagem de leitura, produção oral, produção escrita e sistema de escrita do $2^{\circ}$ ano escolar. É importante destacar que o RAADI possibilita que seja feito o registro considerando-se a qualidade da aprendizagem do estudante da seguinte forma: realiza satisfatoriamente (RS); realiza parcialmente (RP); realiza com ajuda (CA) e não realiza (NR). Os dados foram organizados no Microsoft Excel 2010 e agrupados conforme as necessidades de análise e serão apresentados a seguir.

\footnotetext{
${ }^{1}$ Disponível em SÃO PAULO (2008) ou no sítio da Prefeitura Municipal de Educação de São

Paulo:http://portalsme.prefeitura.sp.gov.br/Documentos/BibliPed/EdEspecial/Referencial_AvaliacaoAprendizagem_ DeficienciaIntelectual.pdf .
} 


\section{Notas Sobre os Resultados}

Iremos, então, apresentar os dados de leitura, produção oral, análise da língua, produção escrita e sistema de escrita, de acordo com os indicadores estabelecidos pelo RAADI. É importante observar que será apresentada a somatória dos dados de todos os estudantes com DI avaliados no $2^{\circ}$ ano escolar. Esses dados se baseiam na avaliação realizada pelas professoras as quais consideraram os níveis de aprendizagem propostos: RS (quando realiza satisfatoriamente), RP (quando realiza parcialmente), CA (quando realiza com ajuda), e NR (quando não realiza). Os dados serão apresentados considerando-se esses níveis de realização pela somatória dos estudantes e poderemos observar suas condições de aprendizagem em grupo e também exemplificaremos alguns casos individuais.

Apresentamos, inicialmente o desempenho geral em Língua Portuguesa, dos 32 estudantes do $2^{\circ}$ ano do ensino fundamental que foram avaliados. Destacamos que no $2^{\circ}$ ano do ensino fundamental, os conteúdos em avaliação referem-se ao que se espera no início do processo de alfabetização, como, por exemplo:

Tabela 1

Exemplificação dos Descritores Avaliados ${ }^{2}$

\begin{tabular}{ll}
\hline Leitura & $\begin{array}{l}\text { Relacionar o gênero à situação concreta de escrita, } \\
\text { reconhecer os suportes textuais mais frequentes da vida } \\
\text { cotidiana, levantar hipóteses de leitura, inferência do } \\
\text { sentido das palavras dentro de um contexto. } \\
\text { Recitar textos memorizados, cantar cantigas de roda } \\
\text { conhecidas, ouvir com compreensão textos contados. }\end{array}$ \\
Produção Oral & $\begin{array}{l}\text { Produção de texto oral, registro de texto coletivo, } \\
\text { escrita de texto de memória, escrever texto de memória } \\
\text { Escrita }\end{array} \quad \begin{array}{l}\text { a partir de suas hipóteses de escrita. } \\
\text { Escrita do próprio nome, reconhecimento das letras e } \\
\text { do alfabeto, localizar palavras conhecidas no texto, } \\
\text { escrever listas, nomes, bilhetes. } \\
\text { Sistema } \\
\text { Alfabética de } \\
\text { Análificar elementos constitutivos do texto (data, } \\
\text { imagens, títulos etc),entre outros descritores. }\end{array}$ \\
\hline
\end{tabular}

Fonte. Elaboração própria.

Os dados estão apresentados em porcentagens e caracterizam o número total de estudantes em avaliação (32 estudantes) e todos os componentes de Língua Portuguesa.

${ }^{2}$ Todos os descritores podem ser acessados em SÃO PAULO, 2008, p. 74 a 76. Foram avaliados 41 descritores no componente de Língua Portuguesa em leitura, produção de escrita, análise da língua, produção oral e sistema de escrita. 


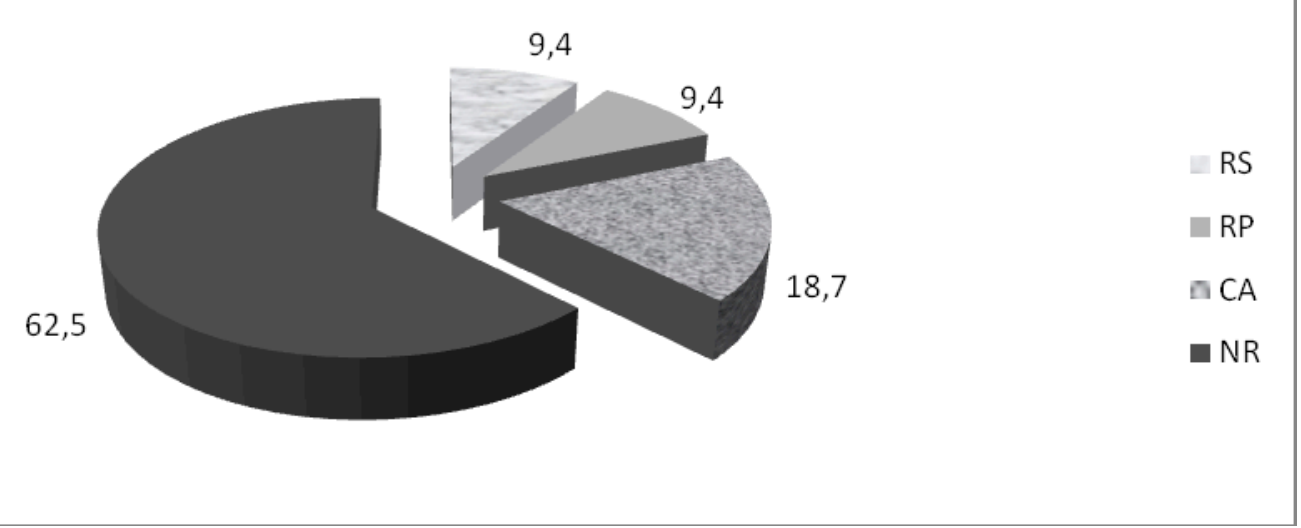

Gráfico 1. Desempenho geral em Língua Portuguesa dos estudantes avaliados.

Como podemos observar no gráfico 1, 62,5\%, correspondendo a 20 dos 32 estudantes são avaliados com dificuldades na realização dos conteúdos gerais da Língua Portuguesa (NR-não realiza), 18,7\% deles (6 estudantes) são capazes de realizar com ajuda (CA), e 9,4\% deles (3 estudantes) realizam satisfatória e parcialmente (RS e RP) os conteúdos avaliados. É uma situação complexa, pois os conteúdos ainda não se referem a uma escrita formal, mas conhecimentos que possibilitam a alfabetização. No entanto, temos que considerar, também, a própria avaliação da professora em relação à deficiência intelectual e o uso do RAADI, que exige um processo de observação e não aplicação de momentos específicos de avaliação, na tentativa de uma avaliação processual do desempenho acadêmico do estudante.

Do mesmo modo, temos que ter cautela na análise, uma vez que é apresentado o conjunto das avaliações de 32 estudantes, porém, se olharmos para cada um deles podemos perceber diferenças importantes. Vejamos alguns exemplos.

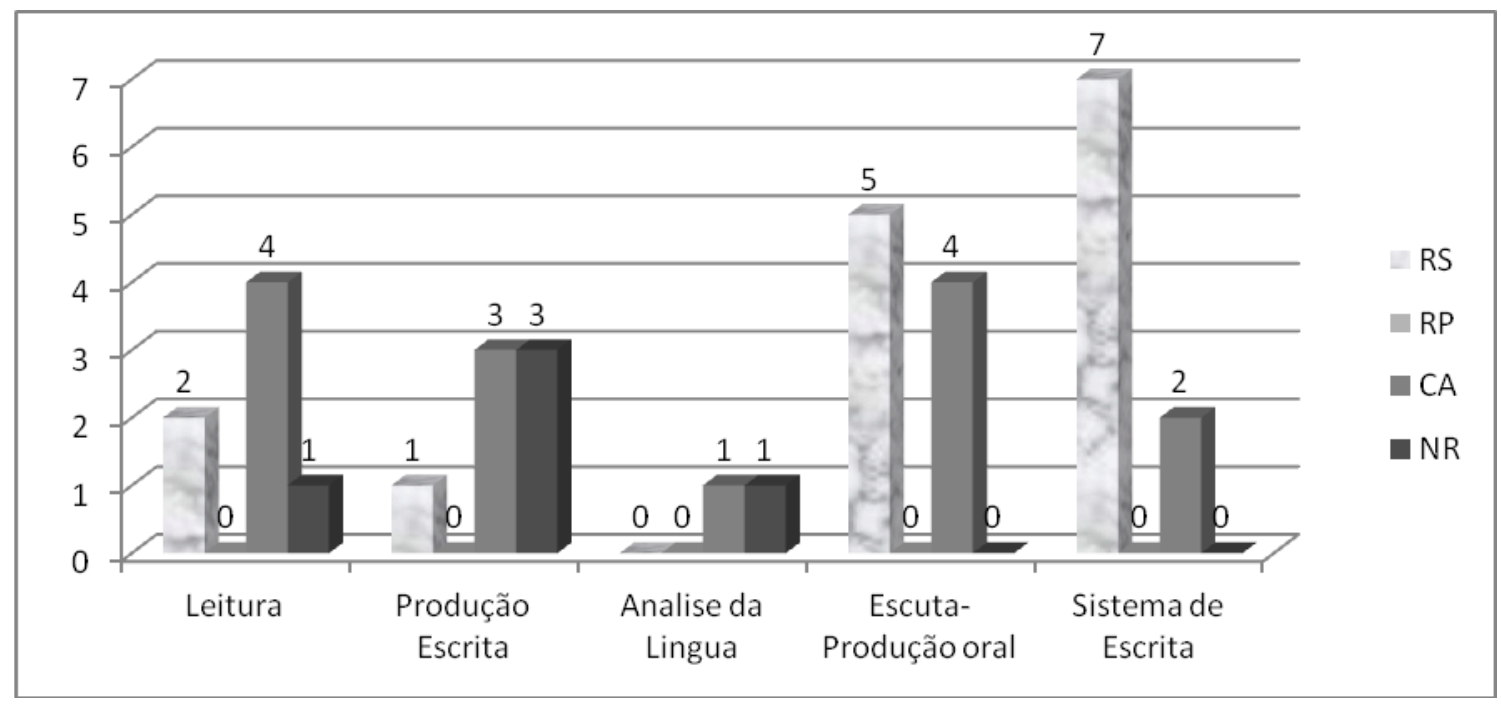

Gráfico 2. Desempenho em LP no $2^{\circ}$ ano do EF do estudante F - Escola A.

No caso do estudante F podemos observar que realiza satisfatoriamente (RS) vários dos conteúdos de Língua Portuguesa, inclusive os de escrita e do sistema de escrita e também apresenta uma realização importante quando se oferece a ajuda (CA). Sua maior dificuldade, conforme o 
gráfico está nos componentes de produção de escrita (NR). De 7 descritores avaliados, não realiza (NR) 3, mas 3 realiza com ajuda (CA) e 1 satisfatoriamente (RS).

A seguir apresentamos o desempenho do estudante $\mathrm{V}$, da escola $\mathrm{L}$ para que possamos observar algumas diferenças de desempenho.

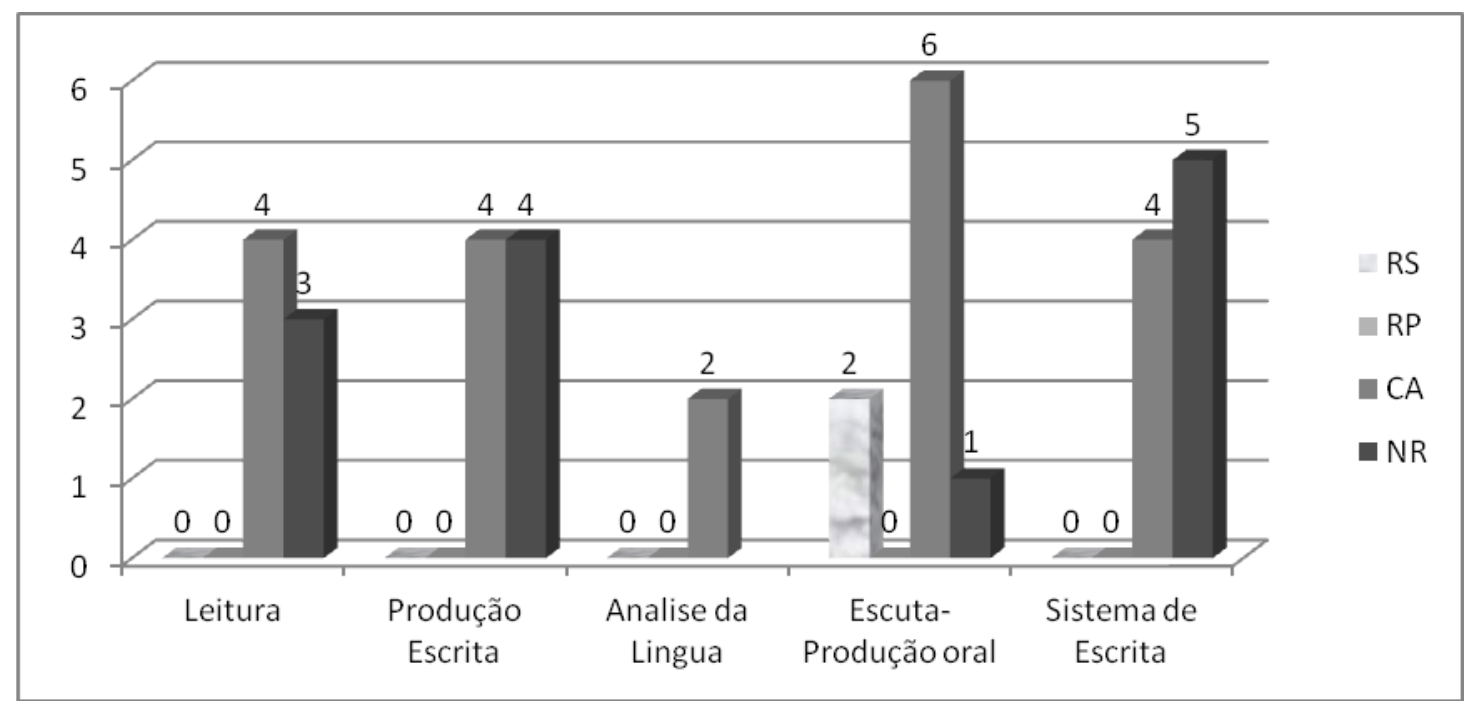

Gráfico 3. Desempenho em LP no $2^{\circ}$ ano do EF do estudante V - Escola F.

Diferentemente da situação do estudante F, o estudante V apresenta maiores dificuldades, o que pode ser observado pelo gráfico, que aponta apenas dois componentes realizados satisfatoriamente (RS) em produção oral, e uma concentração do desempenho com ajuda (CA) na leitura, na escrita, na produção oral e no sistema de escrita, o que é bastante significativo, pois aponta que quando auxiliado suas possibilidades de aprendizagem aumentam, mas também indicam que ainda não tem autonomia plena para alcançar um desempenho satisfatório. Também há mais componentes avaliados como não realiza (NR) do que no caso de F, sendo que $V$ apresenta 13 componentes curriculares que não consegue realizar (NR), com uma ênfase maior na escrita e sistema de escrita.

Também podemos observar o desempenho de $\mathrm{M}$.

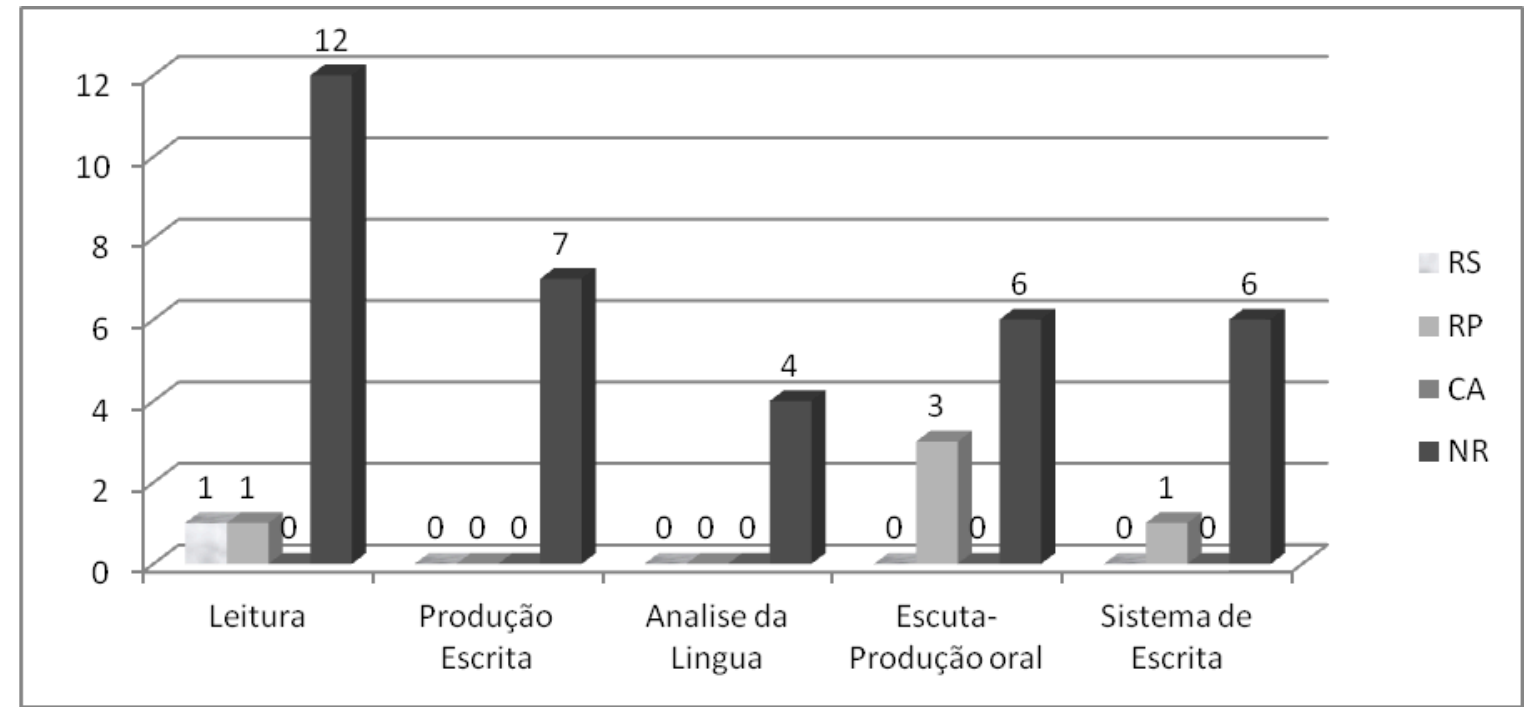

Gráfico 4. Desempenho em LP no $2^{\circ}$ ano do EF do estudante M - Escola J. 
A situação do estudante $M$ também demonstra bastante diferença em relação aos outros, uma vez que seu desempenho, de acordo com a avaliação de sua professora, concentra-se em não realiza (NR), ou seja, de 41 descritores analisados, em 30 é avaliado como com dificuldades para realização, com ênfase na leitura, seguida da produção escrita, sistema de escrita e produção oral. Outro ponto que nos chama a atenção no gráfico 4 é o fato de não ter indicação de realização com ajuda (CA). Qual seria o motivo? Sua professora não considerou essa possibilidade ou ele realmente não muda seu desempenho mesmo que com ajuda?

Bem, o que pudemos perceber é que não podemos perder de vista os desempenhos individuais, mesmo quando estamos realizando uma análise do grupo que se torna importante para que a escola possa ter uma visão do nível de desempenho dos estudantes com DI em cada ano escolar, no sentido de identificar as fragilidades na aprendizagem e tomar providências, ou seja, propor formação continuada aos professores, adquirir novos recursos materiais, reorganizar o planejamento de ensino entre outras ações de cunho administrativo ou político.

Também avaliamos a condição de leitura e escrita separadamente e comparativamente, no âmbito geral, embora, da mesma forma, a análise pode ser realizada individualmente, ou seja, considerando a condição de cada um dos estudantes.

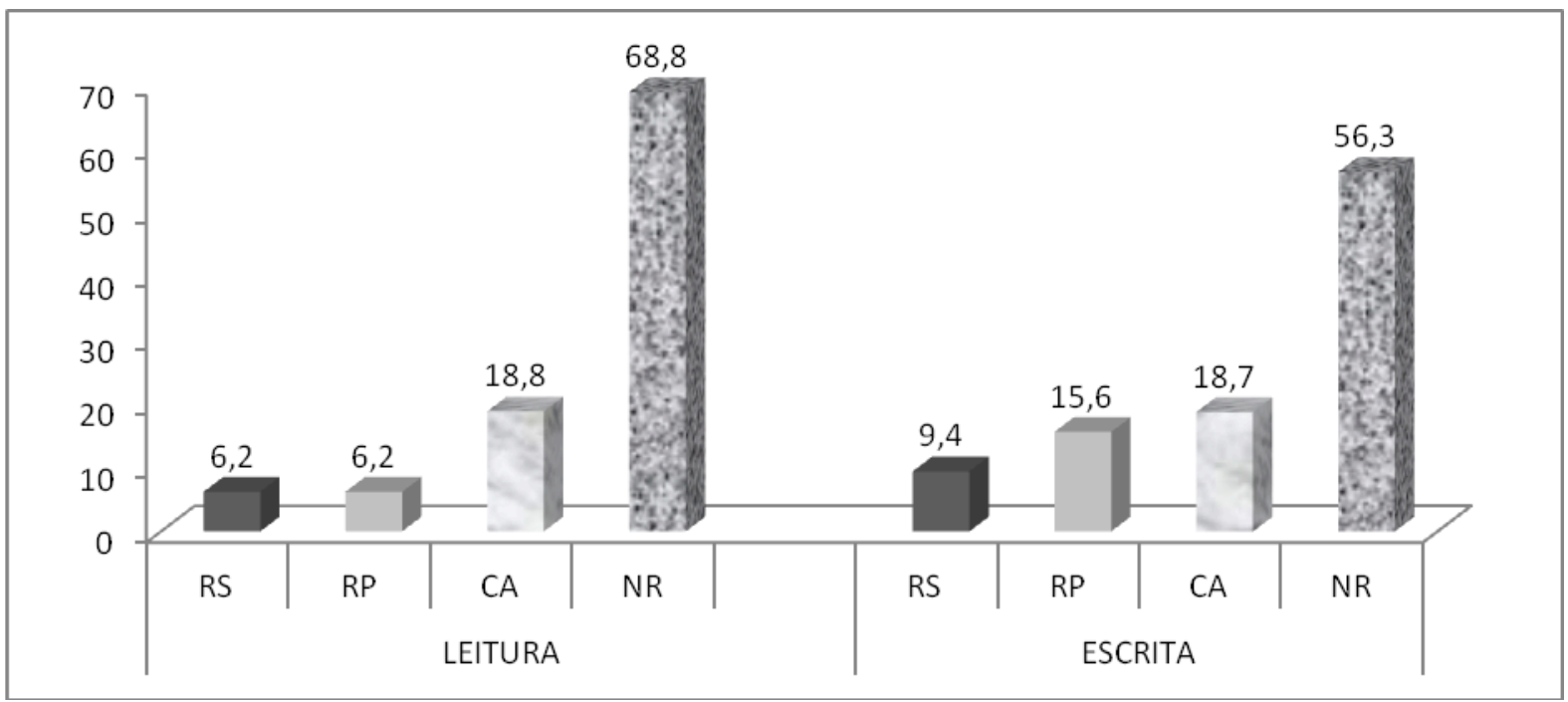

Gráfico 5. Comparação de competência e leitura e escrita de estudantes com DI no $2^{\circ}$ ano do EF.

Diferentemente do que observado em outros estudos (Oliveira, 2011, 2012) estes estudantes apresentam ligeiramente melhor desempenho em escrita do que em leitura. Em relação às dificuldades expressas na não realização (NR) são apontados 56,3\% dos estudantes em escrita, o que equivale a 18 estudantes com dificuldades e 68,8\%, equivalente a 22 estudantes, em leitura. No que se refere à realização satisfatória e parcial (RS e RP), somaríamos $25 \%$, correspondente a 8 estudantes que realizam adequadamente, mesmo que parcial, os descritores de escrita e 12, 4\%, correspondente a 4 estudantes, que o fazem na área da leitura. No item com ajuda (CA) podemos perceber uma aproximação de competência tanto na leitura $(18,8 \%)$ quanto na escrita $(18,7 \%)$.

Um dos fatores a serem assinalados na tentativa de interpretar os resultados é que no $2^{\circ}$ ano do ensino fundamental ainda não se exige a escrita sistemática ou formal, e sim as hipóteses de escrita e a compreensão de alguns elementos do sistema alfabético, como o reconhecimento do alfabeto, de palavras conhecidas no texto, do próprio nome, a produção de texto oral, o estabelecimento de relações entre logotipo e escrita convencional, etc. Na leitura temos descritores 
que exigirão compreensão, reconhecimento de espaços narrativos, recuperação de informações, conhecimento de gêneros, sequência temporal etc., que podem ser aspectos mais difíceis de compreensão por parte destes estudantes.

\section{Considerações Finais}

Avaliar de forma processual ainda é um grande desafio para escola brasileira e o mesmo se pôde observar em relação ao estudante com deficiência intelectual. Além disto, o processo de inclusão traz implicações para a constituição do espaço escolar, exigindo modificações significativas nos processos de ensino em todos os seus âmbitos, do político ao pedagógico.

Neste texto tentamos demonstrar a complexidade do processo de avaliação e mais particularmente quando falamos em deficiência intelectual. O RAADI é um instrumento que nos permite olhar para alguns aspectos dos componentes curriculares utilizando a observação como um importante procedimento de avaliação. Há a intenção de reafirmar a possibilidade de aprendizagem curricular deste estudante, que ao adentrar na escola regular, deve ter garantido o seu direito de desenvolvimento, o qual é impulsionado pela aprendizagem, portanto, pela mediação pedagógica.

Pudemos observar que as dificuldades ainda são significativas - talvez seja também para os estudantes não deficientes -, mas que há possibilidades e que estas devem ser consideradas pelos professores e impulsionadas, na busca de compensar as fragilidades impostas pela deficiência intelectual.

Os resultados encontrados devem ser relativizados, pois aqui estamos olhando particularmente para o estudante e seu desempenho, mas, obviamente, seu desempenho depende em grande medida da ação pedagógica, do fazer docente, do ato de ensino e, podemos inferir o quanto ainda existe de dificuldades no contexto escolar inclusivo para uma prática pedagógica que possa, realmente, impulsionar a aprendizagem daqueles com deficiência intelectual, não porque os professores ou professoras não queiram, mas porque, as condições efetivas de trabalho ainda estão para serem efetivadas, as quais vão desde as providências políticas e administrativas, na construção de materiais e referenciais específicos, na organização da formação continuada e permanente de seus professores; às providências pedagógicas e cotidianas da escola, onde o professor especialista exerce um significativo papel de formador e de suporte para o trabalho qualificado na classe comum, permitindo ao estudante com deficiência intelectual que, da mesma forma que os outros, tenha o acompanhamento de seu processo de aprendizagem e que a escola possa exercer uma análise de suas condições diante do currículo escolar e que, ao conhecê-las, ofereça-lhes o apoio necessário para que também, assim como os outros, possa ter sucesso em sua trajetória escolar, mesmo que siga passos diferentes dos outros estudantes.

\section{Referências}

Boneti, R. V. F. (1999). A interpretação da escrita pela criança portadora de deficiência intelectual. Revista Brasileira de Educação Especial, 3(5), p 41-56.

Braun, P. (2012). Práticas pedagógicas e o estudante com deficiência intelectual: uma intervenção colaborativa sobre os processos de ensino e aprendizagem na perspectiva histórico-cultural. Tese de doutoramento não-publicada. Faculdade de Educação. Universidade Estadual do Rio de Janeiro, Rio de Janeiro: RJ, Brasil.

Brasil. (1996). Lei de Diretrizes e Bases da Educação Nacional: nova LDB (Lei n. 9.394). Rio de Janeiro: RJ, Brasil. Qualitymark

Brasil. (2001). Ministério da Educação. Diretrizes Nacionais para a Educação Especial na Educação Básica. Resolução n. 02/01. Institui as Diretrizes Nacionais para a Educação Especial na Educação Básica. Conselho Nacional de Educação (CNE). Brasília, Brasil. 
Brasil. (2003). Ministério da Educação. Secretaria de Educação Especial. Saberes e práticas da inclusão: avaliação para identificação das necessidades educacionais especiais. Brasília, Brasil: MEC/SEESP.

Brasil. (2010a). Ministério da Educação. Secretaria de Educação Especial. Marcos políticos-legais da Educação Especial na perspectiva da educação inclusiva. Brasília, Brasil: MEC/SEESP.

Brasil. (2010b). Ministério da Educação. Secretaria de Educação Especial. A Escola Comum Inclusiva. Brasília, Brasil: MEC/SEESP/UFC.

Brasil. (2010c). Ministério da Educação. Secretaria de Educação Especial. Atendimento Educacional Especializado para o estudante com deficiência intelectual. Brasília, Brasil: MEC; SEESP.

Brasil. (2011a). Ministério da Educação. Secretaria de Educação Especial Decreto N N $^{\circ}$.611, de 17 de novembro de 2011. Diário Oficial da União. Edição extra. BRASIL: 18 de novembro.

Brasil. (2011b). Ministério da Educação. Secretaria de Educação Especial. Nota Técnica nº 62/2011. Brasil: MEC/SECADI/DPEE. 08 de dezembro.

Ferreira, M. C. C. (2007). A educação escolar de estudantes com deficiência intelectual: pode se viabilizar na perspectiva do letramento? In D. M. Jesus, C. R. Baptista, M. A. S. C.Barreto, S. L. Victor (Orgs.), Inclusão: Práticas pedagógicas e trajetórias de pesquisa. Porto Alegre, Brasil: Mediação.

Gil, A. C. (2002). Como elaborar projetos de pesquisa. São Paulo, country name Atlas. Recuperado em 05 de maio de 2014, de http://www.pedagogia.quirinopolis.ueg.br/abnt/TiposdePesquisaAntonioCarlosGil.pdf.

Glat, R. (Org.). (2007). Educação inclusiva: Cultura e cotidiano escolar. Rio de Janeiro, country name: Sete Letras.

Iacono, J. P. (2012). Língua escrita, deficiência intelectual e cognição: algumas reflexões na perspectiva histórico-cultural. In E. Rossetto,\& D. C.Real. (Orgs.), Diferentes modos de narrar os sujeitos da educação especial a partir de...Cascavel, Pr country name: EDUNIOESTE.

Ide, S. M. (1992) Alfabetização e deficiência mental. Revista Brasileira de Educação Especial, 1(1), 41-50.

Ide, S. M. (1993). Leitura e Escrita e a deficiência mental. São Paulo, Memnon.

Jesus, D. M., Baptista, C. R., Barreto, M. A. S. C., \& Victor, S. L. (Orgs.) (2007). Inclusão: práticas pedagógicas e trajetórias de pesquisa. Porto Alegre, Brasil: Mediação.

Machado, F. A., \& Marquezan, R. (2003). O texto jornalístico: Desencadeador do desenvolvimento da leitura e da escrita da pessoa com deficiência mental. Cadernos de Educação Especial, 1(21), pages?

Mantoan, M. T. E. (1989) Compreendendo a deficiência mental: Novos caminhos educacionais. São Paulo, country name: Scipione.

Monteiro, M. I. B. (2004). A escrita e os estudantes deficientes mentais nas escolas comuns e especiais. In A. C. B. Lodi, K. M. P. Harrison,\& S. R. L. Campos (Orgs.), Leitura e escrita no contexto da diversidade. Porto Alegre, country name: Mediação.

Oliveira, A. A. S., Omote, S., \& Giroto, C. R. M. (Orgs.). (2008). Inclusão Escolar: as contribuições da Educação Especial. São Paulo, Brasil: Cultura Acadêmica Editora.

Oliveira, A. A. S. (2010). Notas sobre a apropriação da escrita por crianças com Síndrome de Down. Cadernos de Educação, Pelotas, 36, 337-359, mai./ago. Recuperado em: 04 fev. 2011, de: http://www.ufpel.edu.br/fae/caduc/downloads/n36/15.pdf.

Oliveira, A. A. S. (2011). Aprendizagem escolar e Deficiência Intelectual: a questão da avaliação curricular. In M. D. Pletsch \& A. Damasceno (Orgs.), Educação especial e inclusão escolar: reflexões sobre o faz̧er pedagógico desde a Educação Infantil até o ensino superior (pp. 10-22). Editora Edur, Rio de Janeiro, RJ, Brasil. 
Oliveira, A. A. S. (2012a) Referencial sobre avaliação da aprendizagem na área da deficiência intelectual. Relatório Técnico-cientifico. Edital MCT/CNPq 14/2009 - Universal. CNPq, (nãopublicado)

Oliveira, A. A. S. (2012b). Aprendizagem de estudantes com deficiência intelectual do ciclo I do Ensino Fundamental: o desempenho em leitura e escrita e a formação de professores. Leitura: teoria e prática suplemento, 1796 - 1807.

Omote, S. (1996). Perspectivas para conceituação de deficiências. In Revista Brasileira de Educação Especial, 2(4), 127-136.

Omote, S. (2008). Diversidade, educação e sociedade Inclusiva. In: Oliveira, A. A. S.; Omote, S.; Giroto, C. R. M. (orgs.)(2008). Inclusão Escolar, as contribuições da Educação Especial (p. 1532). São Paulo, Brasil: Cultura Acadêmica Editora.

Padilha, A. M., Oliveira, A. A. S., \& Silva, L. H. (2014). Práticas pedagógicas na área da deficiência intelectual: O contexto do professor e da sua formação. In A. A. S.Oliveira, R. B. Poker, F. I. W. Oliveira (Eds.), Práticas pedagógicas em educação especial: Em direção a uma escola inclusiva. Universidade de Alcalá: Espanha (no prelo).

Pacheco, J. F. A., Eggertsdóttir, R., \& Marinósson, G.L. (2007). Caminhos para a inclusão: um guia para o aprimoramento da equipe escolar. Porto Alegre: Brasil. Artmed.

Pletsch, M. D. (2010). Repensando a inclusão escolar: diretrizes políticas, práticas curriculares e deficiência intelectual. Rio de Janeiro, RJ, Brasil. Nau: Edur.

Saad, S. N. (2003). Preparando o caminho da inclusão - dissolvendo mitos e preconceitos em relação à pessoa com Síndrome de Down. São Paulo: Vetor.

São Paulo (cidade) Secretaria Municipal de Educação. Diretoria de Orientação Técnica. (2008). Referencial sobre Avaliação da Aprendizagem na área da Deficiência Intelectual. Secretaria Municipal de Educação. São Paulo, SP, Brasil: Secretaria Municipal de Educação / Diretoria de Orientações Técnicas.

Valentim, F. O. D. (2011). Inclusão de estudantes com deficiência intelectual: Considerações sobre avaliação da aprendizagem escolar. Dissertação não-publicada. Faculdade de Filosofia e Ciências, Universidade Estadual Paulista, Marília, SP, Brasil.

Vygotski, L. S. (1997). Fundamentos da Defectologia. Obras Escogidas. Tomo V, Cuba: Editorial Pueblo y Educación.

Vygotski, L. S. (2000). Problemas del desarrollo de la psique - Obras Completas - tomo três. Cuba: Editorial Pueblo y Educación. 


\section{Sobre a Autora}

\section{Anna Augusta Sampaio de Oliveira}

Professora do Programa de Pós-Graduação em Educação, na linha de pesquisa Educação Especial e do Departamento de Educação Especial da Faculdade de Filosofia e Ciências - Universidade Estadual Paulista - UNESP, campus de Marília - SP anna64.oliveira@gmail.com

Professora Assistente Doutora - nível II, da Universidade Estadual Paulista Júlio de Mesquita Filho UNESP, na graduação e pós-graduação. Exerce a função de vice-coordenadora do Programa de Pós-Graduação em Educação, conceito 5 pela CAPES. Doutorado em Educação (Universidade Estadual Paulista Júlio de Mesquita Filho- UNESP, mestrado em Educação Especial (Universidade Federal de São Carlos UFSCar) e graduada em Pedagogia (Universidade de São Paulo- USP). Foi coordenadora de três edições em Curso de Especialização de Formação de Professores em Educação Especial, num convênio junto à Secretaria de Educação do Município de São Paulo e temporariamente do Curso de Especialização em Atendimento Educacional Especializado (AEE), na modalidade a distância, no período de maio de 2009 a novembro de 2010. Também exerceu assessoria pedagógica, na área da Educação Especial, junto à Secretaria de Educação do Município de São Paulo, durante 4 anos. Elaborou um Referencial de Avaliação da Aprendizagem na área da deficiência intelectual, para o Ensino Fundamental, ciclos 1 e 2 e para a Educação de Jovens e Adultos - EJA, e acompanhou a aplicação desse referencial na rede municipal de educação de São Paulo. Atualmente coordena um projeto internacional e interinstitucional envolvendo a Universidade de Havana (Cuba), Universidade Federal Rural do Rio de Janeiro (Rio de Janeiro), a Universidade Cruzeiro do Sul (São Paulo) e a Universidade Estadual Paulista, campus de Marília. É líder do Grupo de Pesquisa em Inclusão Social - GEPIS, em parceria com Maria Candida Soares Del Masso e coordenadora do Grupo de Estudos Políticos e Pedagógicos sobre Inclusão - GEPPI. Tem experiência na área de Educação, com ênfase em Política Educacional, currículo, avaliação, educação especial e educação inclusiva, atuando principalmente nos seguintes temas: concepção de deficiência, práticas pedagógicas inclusivas, avaliação educacional, aprendizagem e formação de professores. Publicou o livro: Um diálogo esquecido: a vez e a voz de adolescentes com deficiência, com várias reedições, organizou o livro: Inclusão escolar: as contribuições da educação especial, em 2008 e reimpressão em 2011, em parceria com Sadao Omote e Claudia Mosca Giroto. Em 2013 publicou o livro Avaliação pedagógica: foco na deficiência intelectual numa perspectiva Inclusiva, em coautoria com Fernanda Dourado Valentim e Luis Henrique Silva e o livro Plano de Desenvolvimento Individual e o Atendimento Educacional Especializado, em coautoria e ambos publicados pela Editora da Unesp. É autora de vários capítulos de livro, artigos nacionais e internacionais, discutindo os temas de sua atuação.

\section{Sobre as Editoras Convidadas}

\section{Márcia Denise Pletsch}

Professora do Programa de Pós-Graduação em Educação, Contextos Contemporâneos e Demandas Populares (PPGEduc) na linha de pesquisa Estudos Contemporâneos e Práticas Educativas e do Departamento Educação e Sociedade da Universidade Federal Rural do Rio de janeiro marciadenisepletsch@gmail.com

A autora é pesquisadora na área de Educação Especial, atuando na formação de professores e de novos pesquisadores. É líder do Grupo de Pesquisa (CNPq) Observatório de Educação Especial e inclusão escolar: práticas curriculares e processos de ensino e aprendizagem e, por meio de convênio 
interinstitucional entre a UFRRJ e a Universidade do Estado do Rio de Janeiro (UERJ), também é líder do grupo de pesquisa Inclusão e aprendizagem de estudantes com necessidades educacionais especiais: práticas pedagógicas, cultura escolar e aspectos psicossociais. Atualmente, coordena o Programa Observatório da Educação da CAPES com projeto de pesquisa em rede na área de deficiência intelectual envolvendo a Universidade Estadual de Santa Catarina (UDESC), Universidade do Vale do Itajaí (UNIVALI); e coordena também pesquisas financiadas pela FAPERJ na área de deficiência múltipla. É autora do livro "Repensando a inclusão escolar: diretrizes políticas, práticas curriculares e deficiência intelectual" e, em colaboração com Rosana Glat, do livro "Inclusão escolar de estudantes com necessidades especiais". Organizou em parceria com outros pesquisadores, entre outros, os livros "Estratégias educacionais diferenciadas para estudantes com necessidades especiais" e "Educação Especial e inclusão escolar: reflexões sobre o fazer pedagógico". Tem mais de vinte artigos publicados em revistas científicas nacionais e internacionais.

\section{Geovana Mendonça Lunardi Mendes}

Professora do Programa de Pós-Graduação em Educação e do Departamento de Pedagogia da Universidade do Estado de Santa Catarina (PGE-UDESC) geolunardi@gmail.com A autora é pesquisadora na área de Educação Especial e dos Estudos Curriculares. Realizou PósDoutorado na Argentina e nos Estados Unidos da América, na área de Currículo e Novas Tecnologias, na Universidad de San Andres em Buenos Aires e em Ashland University, em Ohio. É pesquisadora coordenadora de diferentes projetos de investigação e participa como pesquisadora convidada em projetos de pesquisa nacionais e internacionais. Suas pesquisas e produções têm sido voltadas para área de Currículo e práticas escolares, em especial, as questões relativas as mudanças, novas tecnologias e inovações curriculares no espaço escolar, e também as práticas curriculares voltadas a inclusão de sujeitos com deficiência. Atualmente é a Coordenadora Nacional do Consórcio "Educação e Diversidade" do programa CAPES. FIPSE de Cooperação Internacional, envolvendo a Universidade Federal Rural do Rio de Janeiro, no Brasil e Georgetown College, Ashland University e Brighman Young University nos Estados Unidos e também do Projeto de Pesquisa: Aulas conectadas: mudanças curriculares e aprendizagem colaborativa nas escolas do PROUCA em Santa Catarina, com financiamento do CNPq e do Projeto Observatório de Práticas Escolares com financiamento da FAPESC. É coordenadora do Programa de Pós-graduação em Educação, Mestrado e Doutorado, da FAED, UDESC. Coordena o Observatório da Educação: Tablets, Computadores e Laptops, aprovado no Edital OBEDUC/CAPES. Entre suas atuais produções podem ser destacadas o livro intitulado "Objetos Pedagógicos: uma experiência inclusive em oficinas de Artes", em parceria com mais duas autoras e a organização do Livro "Deficiência e Escolarização: novas perspectivas de analise", hoje na segunda edição. É autora de inúmeros capítulos e artigos publicados em periódicos. 


\section{DOSSIÊ
Educação Especial: diferenças, currículo e processos de ensino e
aprendizagem II arquivos analíticos de políticas educativas}

Volume 23 Número $31 \quad 16$ de março de 2015

ISSN 1068-2341

\section{(c)}

SORERIGHISRESERNED O Copyright e retido pelo/a o autor/a (ou primeiro co-autor) que outorga o direito da primeira publicação à revista Arquivos Analíticos de Políticas Educativas. Más informação da licença de Creative Commons encontram-se em http://creativecommons.org/licenses/by-nc-nd/2.5. Qualquer outro uso deve ser aprovado em conjunto pelo/s autor/es e por AAPE/EPAA. AAPE/EPAA é publicada por Mary Lou Fulton Institute Teachers College da Arizona State University. Os textos publicados em AAPE são indexados por CIRC (Clasificación Integrada de Revistas Científicas, Espanha) DIALNET (Espanha),Directory of Open Access Journals, Education Full Text (H.W. Wilson), EBSCO Education Research Complete, , ERIC, , QUALIS A2 (Brasil), SCImago Journal Rank; SCOPUS, SOCOLAR (China). Contribua com comentários e sugestões a http://epaa.info/wordpress/ ou para Gustavo E. Fischman fischman@,asu.edu.

Curta a nossa comunidade EPAA's Facebook https://www.facebook.com/EPAAAAPE e Twitter feed@epaa_aape. 


\section{arquivos analíticos de políticas educativas conselho editorial}

Editor: Gustavo E. Fischman (Arizona State University) Editores Associados: Rosa Maria Bueno Fisher e Luis A. Gandin

(Universidade Federal do Rio Grande do Sul)

Dalila Andrade de Oliveira Universidade Federal de Minas Gerais, Brasil

Paulo Carrano Universidade Federal Fluminense, Brasil

Alicia Maria Catalano de Bonamino Pontificia Universidade Católica-Rio, Brasil

Fabiana de Amorim Marcello Universidade Luterana do Brasil, Canoas, Brasil

Alexandre Fernandez Vaz Universidade Federal de Santa Catarina, Brasil

Gaudêncio Frigotto Universidade do Estado do Rio de Janeiro, Brasil

Alfredo M Gomes Universidade Federal de Pernambuco, Brasil

Petronilha Beatriz Gonçalves e Silva Universidade Federal de São Carlos, Brasil

Nadja Herman Pontificia Universidade Católica -Rio Grande do Sul, Brasil

José Machado Pais Instituto de Ciências Sociais da Universidade de Lisboa, Portugal

Wenceslao Machado de Oliveira Jr. Universidade Estadual de Campinas, Brasil
Jefferson Mainardes Universidade Estadual de Ponta Grossa, Brasil

Luciano Mendes de Faria Filho Universidade Federal de Minas Gerais, Brasil

Lia Raquel Moreira Oliveira Universidade do Minho, Portugal

Belmira Oliveira Bueno Universidade de São Paulo, Brasil

António Teodoro Universidade Lusófona, Portugal

Pia L. Wong California State University Sacramento, U.S.A

Sandra Regina Sales Universidade Federal Rural do Rio de Janeiro, Brasil

Elba Siqueira Sá Barreto Fundação Carlos Chagas, Brasil

Manuela Terrasêca Universidade do Porto, Portugal

Robert Verhine Universidade Federal da Bahia, Brasil

Antônio A. S. Zuin Universidade Federal de São Carlos, Brasil 


\section{education policy analysis archives editorial board}

Editor Gustavo E. Fischman (Arizona State University)

Associate Editors: Audrey Amrein-Beardsley (Arizona State University), Rick Mintrop, (University of California, Jeanne M. Powers (Arizona State University)

Jessica Allen University of Colorado, Boulder

Gary Anderson New York University

Michael W. Apple University of Wisconsin, Madison

Angela Arzubiaga Arizona State University

David C. Berliner Arizona State University

Robert Bickel Marshall University

Henry Braun Boston College

Eric Camburn University of Wisconsin, Madison

Wendy C. Chi* University of Colorado, Boulder

Casey Cobb University of Connecticut

Arnold Danzig Arizona State University

Antonia Darder University of Illinois, UrbanaChampaign

Linda Darling-Hammond Stanford University

Chad d'Entremont Strategies for Children

John Diamond Harvard University

Tara Donahue Learning Point Associates

Sherman Dorn University of South Florida

Christopher Joseph Frey Bowling Green State University

Melissa Lynn Freeman* Adams State College

Amy Garrett Dikkers University of Minnesota

Gene V Glass Arizona State University

Ronald Glass University of California, Santa Cruz

Harvey Goldstein Bristol University

Jacob P. K. Gross Indiana University

Eric M. Haas WestEd

Kimberly Joy Howard* University of Southern California

Aimee Howley Ohio University

Craig Howley Ohio University

Steve Klees University of Maryland

Jaekyung Lee SUNY Buffalo
Christopher Lubienski University of Illinois, UrbanaChampaign

Sarah Lubienski University of Illinois, UrbanaChampaign

Samuel R. Lucas University of California, Berkeley

Maria Martinez-Coslo University of Texas, Arlington

William Mathis University of Colorado, Boulder

Tristan McCowan Institute of Education, London

Heinrich Mintrop University of California, Berkeley

Michele S. Moses University of Colorado, Boulder

Julianne Moss University of Melbourne

Sharon Nichols University of Texas, San Antonio

Noga O'Connor University of Iowa

João Paraskveva University of Massachusetts, Dartmouth

Laurence Parker University of Illinois, UrbanaChampaign

Susan L. Robertson Bristol University

John Rogers University of California, Los Angeles

A. G. Rud Purdue University

Felicia C. Sanders The Pennsylvania State University

Janelle Scott University of California, Berkeley

Kimberly Scott Arizona State University

Dorothy Shipps Baruch College/CUNY

Maria Teresa Tatto Michigan State University

Larisa Warhol University of Connecticut

Cally Waite Social Science Research Council

John Weathers University of Colorado, Colorado Springs

Kevin Welner University of Colorado, Boulder

Ed Wiley University of Colorado, Boulder

Terrence G. Wiley Arizona State University

John Willinsky Stanford University

Kyo Yamashiro University of California, Los Angeles

* Members of the New Scholars Board 


\section{archivos analíticos de políticas educativas consejo editorial}

Editor: Gustavo E. Fischman (Arizona State University)

Editores. Asociados Alejandro Canales (UNAM) y Jesús Romero Morante (Universidad de Cantabria)

Armando Alcántara Santuario Instituto de Investigaciones sobre la Universidad y la Educación, UNAM México

Claudio Almonacid Universidad Metropolitana de Ciencias de la Educación, Chile

Pilar Arnaiz Sánchez Universidad de Murcia, España

Xavier Besalú Costa Universitat de Girona, España Jose Joaquin Brunner Universidad Diego Portales, Chile

Damián Canales Sánchez Instituto Nacional para la Evaluación de la Educación, México

María Caridad García Universidad Católica del Norte, Chile

Raimundo Cuesta Fernández IES Fray Luis de León, España

Marco Antonio Delgado Fuentes Universidad Iberoamericana, México

Inés Dussel FLACSO, Argentina

Rafael Feito Alonso Universidad Complutense de Madrid, España

Pedro Flores Crespo Universidad Iberoamericana, México

Verónica García Martínez Universidad Juárez Autónoma de Tabasco, México

Francisco F. García Pérez Universidad de Sevilla, España

Edna Luna Serrano Universidad Autónoma de Baja California, México

Alma Maldonado Departamento de Investigaciones Educativas, Centro de Investigación y de Estudios Avanzados, México

Alejandro Márquez Jiménez Instituto de Investigaciones sobre la Universidad y la Educación, UNAM México

José Felipe Martínez Fernández University of California Los Angeles, USA
Fanni Muñoz Pontificia Universidad Católica de Perú

Imanol Ordorika Instituto de Investigaciones Economicas - UNAM, México

Maria Cristina Parra Sandoval Universidad de Zulia, Venezuela

Miguel A. Pereyra Universidad de Granada, España

Monica Pini Universidad Nacional de San Martín, Argentina

Paula Razquin UNESCO, Francia

Ignacio Rivas Flores Universidad de Málaga, España

Daniel Schugurensky Arizona State University

Orlando Pulido Chaves Universidad Pedagógica Nacional, Colombia

José Gregorio Rodríguez Universidad Nacional de Colombia

Miriam Rodríguez Vargas Universidad Autónoma de Tamaulipas, México

Mario Rueda Beltrán Instituto de Investigaciones sobre la Universidad y la Educación, UNAM México

José Luis San Fabián Maroto Universidad de Oviedo, España

Yengny Marisol Silva Laya Universidad Iberoamericana, México

Aida Terrón Bañuelos Universidad de Oviedo, España

Jurjo Torres Santomé Universidad de la Coruña, España

Antoni Verger Planells University of Amsterdam, Holanda

Mario Yapu Universidad Para la Investigación Estratégica, Bolivia 